\title{
Cellulose acetate/poly(methyl methacrylate) interpenetrating networks: synthesis and estimation of thermal and mechanical properties
}

\section{$\operatorname{AUTHOR}(\mathrm{S})$ :}

Aoki, Dan; Teramoto, Yoshikuni; Nishio, Yoshiyuki

\section{CITATION:}

Aoki, Dan ...[et al]. Cellulose acetate/poly(methyl methacrylate) interpenetrating

networks: synthesis and estimation of thermal and mechanical properties. Cellulose 2011, 18(6): 1441-1454

\section{ISSUE DATE:}

2011-12

URL:

http://hdl.handle.net/2433/151708

\section{RIGHT:}

The final publication is available at www.springerlink.com; This is not the published version. Please cite only the published version.; この論文 は出版社版でありません。引用の際には出版社版をご確認ご利用くだ さい。 
Cellulose acetate/poly(methyl methacrylate) interpenetrating networks: synthesis and estimation of thermal and mechanical properties

Dan Aoki, Yoshikuni Teramoto, and Yoshiyuki Nishio*

Division of Forest and Biomaterials Science, Graduate School of Agriculture, Kyoto University, Sakyo-ku, Kyoto 606-8502, Japan

* To whom correspondence should be addressed.

E-mail: ynishio@kais.kyoto-u.ac.jp. Tel.: +81 75753 6250. Fax: +81 757536300. 


\section{Abstract}

IPN-type composites consisting of cellulose acetate (CA) and poly(methyl methacrylate) (PMMA) were successfully synthesized in film form. In this synthesis, a mercapto group (SH)-containing CA, CA-MA, was prepared in advance by esterification of CA with mercaptoacetic acid, and then intercomponent cross-linking between CA-MA and PMMA was attained by thiol-ene polymerization of methyl methacrylate (MMA) onto the CA-MA substrate. For comparison, polymer synthesis was also attempted to produce a semi-IPN type of composites comprising CA and cross-linked PMMA, via copolymerization of MMA and ethylene glycol dimethacrylate as cross-linker in a homogeneous system containing CA solute. Thermal and mechanical properties of thus obtained polymer composites were investigated by differential scanning calorimetry, dynamic mechanical analysis, and a tensile test, in correlation with the mixing state of the essentially immiscible cellulosic and methacrylate polymer components. It was shown that the specific IPN technique using thiol-ene reactions usually resulted in a much better compatibility-enhanced polymer composite, which exhibited a higher tensile strength and even an outstanding ductility without parallel in any film sample of CA, PMMA, and their physical blends.

Keywords cellulose acetate; poly(methyl methacrylate); composites; interpenetrating network (IPN); thiol-ene polymerization 


\section{Introduction}

Cellulose acetate (CA) is one of the most common cellulose derivatives because of the scale and multiplicity of uses based on the desirable properties such as good optical clarity in film form, a relatively high modulus and adequate flexural and tensile strengths in film and fiber forms, and potential biodegradability as an additional value (Buchanan et al. 1993; Edgar et al. 2001). However, it is difficult to thermally mold CA without any plasticizer, because the glass transition temperature $\left(T_{\mathrm{g}}\right)$ and flowing point are considerably high, where decomposition of the molecular chains can take place noticeably.

For further functionalization and utilization of $\mathrm{CA}$, the incorporation with a flexible polymer at a hyperfine structural level may be a viable approach (Nishio 2006). A few synthetic vinyl polymers, e.g., poly(vinyl phenol) (Landry et al. 1994), and poly( $N$-vinyl pyrrolidone) and related copolymers (Miyashita et al. 2002; Ohno et al. 2005), have been reported to act as a highly compatible (or miscible) polymeric plasticizer toward CA. However, the variety of such compatible counter-polymers for CA is still limited and most of them are, in common, strongly hydrophilic, and moreover, the blending with CA offers a rather low-strength material as far as the tensile mechanical property is concerned.

A vinyl polymer adopted as a mixing partner for CA in the present work, poly(methyl methacrylate) (PMMA), is also important as optical and medical materials based on its distinguished optical clarity, rigidity, and hydrophobicity. In contrast to CA, PMMA can be molded thermally per se, but the moldings are usually lack of ductility and toughness. An intimate mixture of these two optical ingredient polymers possessing complementary thermal and mechanical characteristics would exhibit improved processibility and wide-ranging physical properties; however, the CA/PMMA pair is immiscible in the thermodynamical sense (Bikson et al. 1994; Ohno and Nishio 2007). Generally, immiscible polymer pairs are accorded less probability to produce some composite material showing preferable properties 
by simple physical blending (Utracki 1990; Nishio 2006).

From a practical standpoint regarding improvement in physical properties of a binary polymer system, the final mixing state of the two components in the actually manufactured product should be the dominant factor. In this sense, even if the polymer pair is originally immiscible, construction of an interpenetrating polymer network (IPN) can be a promising route leading to their compatible composite ensuring enhancement in the performance and/or functionality (Nishio 1994; Nishio 2006). IPN is defined, literally, as a combination of two polymers in network form, at least one of which is usually synthesized from the monomer into macromolecular chains, often cross-linked, in the immediate presence of the other (Thomas and Sperling 1978). In cellulose chemistry, too, this IPN approach has demonstrated an availability for designing novel composites based on the natural polysaccharide (Chang et al. 2009; Miyashita et al. 1998; Nishio and Hirose 1992; Williamson et al. 1998; Yano et al. 2005).

In a preceding paper (Aoki et al. 2007), the authors prepared a series of CA derivatives having a cross-linkable mercapto group, CA-MAs, by esterification of CA with mercaptoacetic acid. The CA-MA samples showed a sol-gel transition in solution and a shape memory-recovery behaviour in film form through adequate redox treatments, due to the reversible, cross-linking association and dissociation between the introduced SH groups. In this present paper, the major objective is to exemplify the successful construction of an IPN-type composite in film form of the immiscible CA and PMMA pair, as further application of the functional mercapto group. For comparative examination, attempts are made to synthesize various network architectures (Figure 1), mutually different in cross-linking situation of the components, but, in common, via polymerization of methyl methacrylate (MMA) in a solution system containing CA or CA-MA solute. The corresponding three representative composites are coded as follows:

- CA/PMMA-e, both polymer components having no chemical cross-linkage, but 
well-entangled with each other (Figure 1a);

- CA/PMMA-EG, only the PMMA component being cross-linked with the aid of ethylene glycol dimethacrylate (EGDM) as cross-linker (Figure 1b);

- CA-MA/PMMA, having an intercomponent cross-linkage generated by thiol-ene polymerization (Kharasch et al. 1938) of MMA onto the SH-containing CA (Figure 1c). This composite series involves, more or less, a structural character resembling that of graft copolymers of cellulosics.

In Figure 1, cross-linking junctions originating from EGDM and mercapto moieties are displayed by filled and open circles, respectively. Thermal and mechanical properties of the three sorts of composites prepared in anticipation of the respective IPN fashions are investigated by differential scanning calorimetry (DSC), dynamic mechanical analysis (DMA), and a tensile test. Main discussion is focused on the network formation and the mixing state of the two polymer components in the composite films.

\section{$<<$ Figure 1 $>>$}

\section{Experimental}

Materials

A CA sample, kindly supplied by Daicel Chemical Industries, Ltd., was purified by dissolving in acetone, reprecipitating into distilled water, and washing with ethanol. The degree of acetyl substitution $\left(\mathrm{DS}_{\mathrm{Ac}}\right.$ ) of the purified CA was 2.28, determined by ${ }^{1} \mathrm{H}$ NMR measurements (see below). Methyl methacrylate (MMA) and mercaptoacetic acid were purchased from Nacalai Tesque, Inc. and distilled before use. Solvents, $N, N$-dimethylformamide (DMF), 1,3-dimethyl-2-imidazolidinone (DMI), and pyridine, were purchased from Nacalai Tesque, Inc. and stored over molecular sieves 4A before use. Propionic anhydride, 
4-(dimethylamino)pyridine, 2,2-dimethoxy-2-phenylacetophenone (DMPA), tetrahydrofuran (THF), ethylene glycol dimethacrylate (EGDM), 2-mercaptoethanol, and other reagents were purchased from Wako Pure Chemical Industries, Ltd. and used as received. DMI was deaerated under reduced pressure and bubbled with $\mathrm{N}_{2}$ gas before use.

CA-MA was newly prepared by esterification of CA with mercaptoacetic acid according to the same procedure as that described previously (Aoki et al 2007). The yield of product was 80-90 wt\%. Substitution parameters and molecular weights of the CA-MA and starting CA samples were determined by ${ }^{1} \mathrm{H}$ NMR and gel permeation chromatography (GPC), respectively, after propionylation of residual hydroxyls (for CA and CA-MA) and terminal mercapto groups (for CA-MA); the experimental detail has been written in the preceding paper (Aoki et al 2007). The chemical shifts observed for the propionylated CA and CA-MA were as follows.

Propionylated CA, ${ }^{1} \mathrm{H}$ NMR $\left(\mathrm{CDCl}_{3}\right): \delta=0.95-1.30\left(3 \mathrm{H}, \mathrm{m}\right.$, propionyl $\left.\mathrm{CH}_{3}\right), 1.82-2.55$ (3H/2H, m, acetyl $\mathrm{CH}_{3}$ /propionyl $\left.\mathrm{CH}_{2}\right), 3.20-5.50$ (7H, m, glucopyranose).

Propionylated CA-MA, ${ }^{1} \mathrm{H}$ NMR $\left(\mathrm{CDCl}_{3}\right): \delta=0.95-1.30\left(3 \mathrm{H}, \mathrm{m}\right.$, propionyl $\left.\mathrm{CH}_{3}\right)$, 1.82-2.55 (3H/2H, m, acetyl $\mathrm{CH}_{3} /$ propionyl $\left.\mathrm{CH}_{2}\right), 2.55-2.80\left(2 \mathrm{H}\right.$, s, thiopropionyl $\left.\mathrm{CH}_{2}\right)$, 3.20-3.95 (2H/2H, m, mercaptoacetyl $\mathrm{CH}_{2} /$ glucopyranose $\mathrm{C} 4-\mathrm{H}$ and $\left.\mathrm{C} 5-\mathrm{H}\right), 3.95-5.50$ (5H, m, glucopyranose C1-H, C2-H, C3-H, C6- $\mathrm{H}_{\mathrm{a}}$, and C6- $\mathrm{H}_{\mathrm{b}}$ ).

Table 1 summarizes data of the determinations of $\mathrm{DS}_{\mathrm{Ac}}$, the degree of substitution (DS) of side chains having a mercapto terminal $\left(\mathrm{DS}_{\mathrm{SH}}\right)$, the molar substitution of mercaptoacetic acid $\left(\mathrm{MS}_{\mathrm{MA}}\right)$, the propionyl DS $\left(\mathrm{DS}_{\mathrm{Pr}}\right)$, the number-average molecular weight $\left(M_{\mathrm{n}}\right)$, the weight-average molecular weight $\left(M_{\mathrm{w}}\right)$, and the degree of polydispersity $\left(M_{\mathrm{w}} / M_{\mathrm{n}}\right)$ for the CA and CA-MA samples employed for composite synthesis in this study. Hereafter CA-MA of $\mathrm{DS}_{\mathrm{SH}}=x$ is designated as CA-MA $x$

\section{$<<$ Table 1 $>>$}

PMMA homopolymer was synthesized by radical polymerization of MMA with 
ultraviolet (UV) irradiation. $10 \mathrm{~g}$ of MMA containing a photo initiator DMPA at a concentration of $0.5 \mathrm{wt} \%$ was dissolved in $70 \mathrm{~g}$ of DMI. The radical polymerization was conducted at $20{ }^{\circ} \mathrm{C}$ for $3 \mathrm{~h}$ under a nitrogen atmosphere in a curing chamber equipped with a 10 W UV lamp FL10BLB-A (Toshiba Lighting \& Technology Corporation). Subsequently, after taking off the UV lamp, the solution was heat treated at $80{ }^{\circ} \mathrm{C}$ for $3 \mathrm{~h}$ under an $\mathrm{N}_{2}$ infusion. Following that, a PMMA solid bulk was obtained by solvent evaporation of the solution at $50{ }^{\circ} \mathrm{C}$ under reduced pressure. The crude PMMA material was dissolved in THF, reprecipitated in an excess amount of distilled water, and filtered. Finally, the product was thoroughly dried at $40{ }^{\circ} \mathrm{C}$ for $120 \mathrm{~h}$ in a vacuum oven.

Preparation of Cast Films of CA, PMMA, and Their Blends

CA and PMMA solutions in DMF were separately prepared at a polymer concentration of $5 \mathrm{wt} \%$, and aliquots of the two solutions were mixed with each other in the desired proportions. The respective solutions were poured into a Teflon tray and film samples of CA, PMMA, and their blends (designated as CA/PMMA- $b$ ) were made by solvent evaporation at $40{ }^{\circ} \mathrm{C}$ under reduced pressure. The as-cast films were dried at $50{ }^{\circ} \mathrm{C}$ for $120 \mathrm{~h}$ in a vacuum oven. Blend films were also made from similarly mixed solutions in DMI instead of DMF, but we found no significant difference in thermal property between the two cast series.

Preparation of IPN-type Composite Films

Three series of composites, CA/PMMA-e, CA/PMMA-EG, and CA-MA/PMMA, were synthesized by radical polymerization of MMA in DMI dissolving CA or CA-MA. The MMA monomer contained DMPA as photo-initiator at $0.5 \mathrm{wt} \%$, and the concentration of CA or CA-MA in DMI was adjusted to $2.8 \mathrm{wt} \%$ before addition of the monomer therein. In 
preparation of CA/PMMA-EG, a prescribed amount of EGDM was also fed into the CA solution, to attain cross-linking of the resulting PMMA component. Table 2 tabulates in-feed amounts of the reagents required for concrete preparations of the three series. The respective homogeneous mixtures were poured into a Teflon tray and the polymerization reaction was carried out in a manner similar to that adopted for the PMMA homopolymer synthesis, i.e., with UV irradiation for $3 \mathrm{~h}$ at $20{ }^{\circ} \mathrm{C}$ and heat treatment for $3 \mathrm{~h}$ at $80{ }^{\circ} \mathrm{C}$, both under a nitrogen atmosphere. The solutions for preparing CA/PMMA-EG and CA-MA/PMMA samples transformed into a gel (still transparent) in the course of UV irradiation, indicating appreciable development of cross-links, but the other reacting system leading to CA/PMMA-e did not gelate on the exterior. By solvent evaporation of the gels and solution at $50{ }^{\circ} \mathrm{C}$ under reduced pressure, composite samples were cast into a film several hundreds of micrometers thick. The as-cast composite films were washed with distilled water and dried at $50{ }^{\circ} \mathrm{C}$ for $120 \mathrm{~h}$ in a vacuum oven. The thoroughly dried samples were supplied for visual inspection, and quantifications of the actual weight ratio of the two constituent polymers and the molecular weight of the PMMA component, in advance of the detailed thermal and mechanical characterizations.

\section{$<<$ Table 2 $>>$}

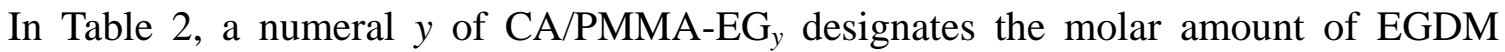
in-fed to synthesize the composite concerned, paralleling the value of $\mathrm{DS}_{\mathrm{SH}}$ denoted by $x$ for the CA-MA/PMMA series. For example, the in-feed amount of EGDM regarding a $\mathrm{CA} / \mathrm{PMMA}-\mathrm{EG}_{0.21} \# 2$ synthesis was equalized to the mole number of $\mathrm{SH}$ groups as to

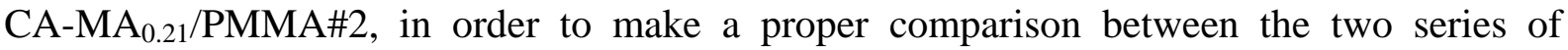
composites. The amounts of MMA to be weighed were determined based on a preliminary data on the conversion rate of the monomer to polymer in solution with DMI, so that we could obtain various weight ratios of CA:PMMA: \#1, 20:80; \#2, 50:50; and \#3: 80:20. 
PMMA Extraction from Composite Films

For the purpose of estimating the molecular weight of the PMMA component in the three series of composites, the polymer extraction was performed by using THF. $0.5 \mathrm{~g}$-Fragments cut out from each composite film were added into THF $(10 \mathrm{~mL})$ and the mixture was stirred at $25^{\circ} \mathrm{C}$ for $96 \mathrm{~h}$ and filtered. The filtrate, containing extracted PMMA that was assumed to be an originally uncross-linked fraction, was examined for evaluations of $M_{\mathrm{n}}$ and $M_{\mathrm{w}}$ by GPC analysis.

With regard to the CA-MA/PMMA series, especially for the THF-insoluble part filtered off, a base treatment was carried out to separate the chemically grafted PMMA chains from the cellulose trunk and extract them, in the following way: A 0.2-g weight of the dried THF-insoluble residue was suspended and stirred in $30 \mathrm{~mL}$ of $2 \mathrm{M} \mathrm{NaOH}$ aqueous solution at $70{ }^{\circ} \mathrm{C}$. After $48 \mathrm{~h}$, the suspension was cooled to $25^{\circ} \mathrm{C}$ and mixed with $60 \mathrm{~mL}$ of aqueous $\mathrm{HCl}$ (1 M), followed by stirring for another $2 \mathrm{~h}$. A solid mixture of free PMMA and cellulose (restored by deesterification) was filtered off and dried at $50{ }^{\circ} \mathrm{C}$ overnight in a vacuum oven. Again, extraction of the PMMA fraction from the polymer mixture was conducted with THF, and a PMMA/THF solution obtained as filtrate was used for the molecular weight quantification by GPC. For the cellulose residue, the deesterified state was confirmed by infra-red spectroscopic identification, and, more facilely, by microscopic Raman spectroscopy; decisively, a specific band centering $\sim 1750 \mathrm{~cm}^{-1}$ derived from $\mathrm{C}=\mathrm{O}$ stretching vibrations disappeared after the hydrolytic base-treatment, proving the eliminations of the acetyl and mercaptoacetyl groups as well as of the grafted PMMA chains from the CA derivative.

Reduction treatment of CA-MA/PMMA Composite Films 
To confirm the presence of disulfide cross-linking in CA-MA/PMMA films, a reduction treatment was performed by using 2-mercaptoethanol that dissociates $\mathrm{S}=\mathrm{S}$ into $\mathrm{SH}$ groups (Aoki et al. 2007). 0.1 g-Fragments cut out from each composite film were added into DMI (2 g) and the mixture was stirred at $25^{\circ} \mathrm{C}$ for $24 \mathrm{~h}$. Subsequently, 2-mercaptoethanol $(1 \mathrm{~g})$ was added into the mixture, followed by stirring at $25{ }^{\circ} \mathrm{C}$ for $24 \mathrm{~h}$. The mixture was then reprecipitated in an excess amount of distilled water, washed with methanol, and filtered. Finally, the product was dried at $40{ }^{\circ} \mathrm{C}$ for $48 \mathrm{~h}$ in a vacuum oven.

\section{Measurements}

500-MHz ${ }^{1} \mathrm{H}$ NMR spectra were measured to determine DS values for different cellulose ester derivatives by using a Varian NMR system $500 \mathrm{MHz}$ with an OneNMR 5MM Probe. The measuring conditions were as follows: solvent, $\mathrm{CDCl}_{3}$; solute concentration, $20 \mathrm{mg} \mathrm{mL}{ }^{-1}$; internal standard, tetramethylsilane; temperature, $23{ }^{\circ} \mathrm{C}$; number of scan, 64 ; recycle time of pulse, $6.5 \mathrm{~s}$.

GPC analysis was carried out with a Tosoh HLC-8220 GPC apparatus. The measuring conditions were as follows: column, two Tosoh TSK Super HZM-H columns connected with each other; flow rate, $0.25 \mathrm{~mL} \mathrm{~min}{ }^{-1}$; temperature, $40{ }^{\circ} \mathrm{C}$; eluent, THF; standard, monodispersed polystyrene.

Raman spectra were measured by using a Horiba LabRam-350V microscopic Raman spectroscopy apparatus on the following condition: number of scan, 64; magnification, 50 times.

DSC was carried out with a Seiko DSC6200/EXSTAR6000 apparatus. The temperature readings were calibrated with an indium standard. The measurements were made on ca. 4 mg of samples packed in an aluminum pan, usually at a scanning rate of $20{ }^{\circ} \mathrm{C} \mathrm{min}{ }^{-1}$ under a nitrogen atmosphere. Preliminarily, each individual sample was heated from 25 to $238{ }^{\circ} \mathrm{C}$ 
and held at the temperature for 3 min. Successively, the sample was cooled to $-50{ }^{\circ} \mathrm{C}$ at a rate of $80{ }^{\circ} \mathrm{C} \min ^{-1}$. Following this, the second heating scan was run over the temperature range -50 to $240{ }^{\circ} \mathrm{C}$. The glass transition temperature $T_{\mathrm{g}}$, estimated in the second heating scan, was taken as a temperature at the midpoint of the discontinuity in heat flow.

DMA was conducted by using a Seiko DMS6100/EXSTAR6000 apparatus. Film strips of rectangular shape $\left(30 \times 5 \mathrm{~mm}^{2}\right)$ were used for measurements of the temperature dependence of the dynamic storage modulus $E^{\prime}$, loss modulus $E^{\prime \prime}$, and mechanical loss tangent $\tan \delta$. Before the measurements, the samples were sandwiched between hard card-boards and heat treated at $170{ }^{\circ} \mathrm{C}$ for $5 \mathrm{~min}$ in a vacuum oven, to remove trace amounts of solvent and relax possible stresses. The measuring conditions were as follows: temperature range, 30-260 ${ }^{\circ} \mathrm{C}$; scanning rate, $2{ }^{\circ} \mathrm{C} \min ^{-1}$; oscillatory frequency, $10 \mathrm{~Hz}$. All the measurements were duplicated, and there was no substantial difference between the DMA data for two specimens cut from the same film prepared in a larger size.

A tensile mechanical test was conducted by using a Shimadzu Autograph AGS-5kNG apparatus, to evaluate a Young's modulus, yield stress, elongation at rupture, and tensile strength. Film strips $\left(30 \times 5 \mathrm{~mm}^{2}\right)$ were used for the measurements. Prior to the test, the samples were heat treated at $170{ }^{\circ} \mathrm{C}$ for $5 \mathrm{~min}$ in a vacuum oven and then settled at $23{ }^{\circ} \mathrm{C}$ and relative humidity $50 \%$ for $48 \mathrm{~h}$. The measuring conditions were as follows: temperature, $23{ }^{\circ} \mathrm{C}$; relative humidity, $50 \%$; gauge length, $15 \mathrm{~mm}$; elongation rate, $0.1 \mathrm{~mm} \min ^{-1}$. Five strips were cut from a given larger film and average values were adopted for the tensile data reported.

\section{Results and Discussion}

General Features of Composite Films 
Chemical synthesis of three series of composites, CA/PMMA-e, CA/PMMA-EG, and CA-MA/PMMA, were accomplished according to the experimental procedure described above. A weight ratio of the two major polymer components in the composites was calculated based on the weight yield of the respective film products, by assuming that the in-feed quantity of CA (or CA-MA) was carried over in the entirety into each solid matrix. The result is listed in Table 3. The table also includes a brief description of visual appearance for the composite films, and for films of physical blends (CA/PMMA- $b$ ) as well.

\section{$\langle<$ Table 3 $>$}

Film samples of the CA/PMMA- $b$, CA/PMMA- $e$, and CA/PMMA-EG series were all visually turbid, more or less; this is ascribable principally to poor miscibility of the polymer pair (Bikson et al. 1994; Ohno and Nishio 2007), but there is partly a possibility of arising from development of micro-voids. Strictly, CA/PMMA-e and CA/PMMA-EG samples were uniformly turbid, whereas CA/PMMA- $b$ ones had more or less polka dots of a few millimeters in diameter. Apparently, the scale of phase separation in the two composite series may be taken as being smaller, compared with the physical blends showing such a macroscopic, sea-island-type segregation. It can therefore be said that the method of incorporating PMMA with CA by vinyl polymerization downsizes the inherent mixing scale of the two components to a certain extent.

In contraposition, all samples of the other series CA-MA/PMMA were obtained as a transparent film, irrespective of the $\mathrm{DS}_{\mathrm{SH}}$ of $\mathrm{CA}-\mathrm{MA}$ in-fed and the ingredient composition preset. Incidentally, casting of a solution containing CA-MA and separately polymerized PMMA resulted in formation of a phase-separated film similar to the CA/PMMA- $b$ samples. These observations suggest attainment of an intimate mixing of the CA and PMMA components via thiol-ene grafting, the homogeneity scale being smaller than the wavelength order of visible light. In this connection, through consumption of the initial mercapto groups 
of CA-MA in the composite synthesis was confirmed by Raman spectroscopic measurements; that is, a peak intensity at $2575 \mathrm{~cm}^{-1}$ assigned to the $\mathrm{SH}$ group of high polarizability (Evans and Ellman 1959) vanished after the synthesis. However, a portion of mercapto groups may have been consumed by another reaction, i.e., disulfide cross-linking. To see the presence of the disulfide cross-linking, a reduction treatment was applied to CA-MA/PMMA films with 2-mercaptoethanol. Figure 2 illustrates Raman spectra of CA-MA $0.21 / \mathrm{PMMA \# 2}$ and CA-MA $\mathrm{M}_{0.21} / \mathrm{PMMA \# 3}$ measured after the reduction treatment. In the spectrum of the former sample (\#2), there appeared no distinct peak around $2575 \mathrm{~cm}^{-1}$. On the contrary, the latter one (\#3) provided a peak at $2575 \mathrm{~cm}^{-1}$ assigned to the restored $\mathrm{SH}$ group. This result implies that CA-MA 0.21 PMMA\#3 formed an appreciable amount of disulfide cross-links in the preparation process due to the richness in the CA-MA ingredient.

\section{$<<$ Figure 2 $>>$}

Results of the GPC analysis of extracted PMMA for the three composite series are also listed in Table 3, together with the corresponding data of molecular weights for a plain PMMA sample separately polymerized in DMI. Regarding the PMMA fractions directly extracted from the composites with THF, their molecular weights were generally lower than that of the PMMA homopolymer. This is readily interpretable as due to the conditions of composite synthesis, i.e., a higher viscosity of the reactive system dissolving CA or CA-MA and a lower concentration of the in-feed MMA monomer (see Experimental section). By way of exception, \#1 samples (rich in PMMA) of the CA/PMMA-EG series exhibited rather higher molecular weights associated with the extracted PMMA components. It is presumable that the THF-soluble fractions contained a good deal of PMMA chains slightly cross-linked with EGDM.

In synthesis of the CA-MA/PMMA series, graft-like copolymers of CA with dangling chains of PMMA are produced by thiol-ene polymerization, in competition with occurrence of free PMMA chains by normal radical polymerization. A secondary structure, 
CA-PMMA-CA, can also be generated by radical-coupling termination of two propagating PMMA grafts. Molecular weights of the PMMA graft-chains were evaluated by GPC through the separation and extraction via base-treating for the initially THF-insoluble part of the CA-MA/PMMA samples, as described in the preceding section. A similar base treatment was applied to PMMA per se, and it was confirmed that the average molecular weight was almost unchanged in the treatment process (see the top data in the right column of Table 3), implying no prevalence of the polymer degradation (including elimination of side groups) thereby. As is evident from the result of GPC analysis in Table 3, the molecular weight $\left(M_{\mathrm{n}}\right)$ of the grafted PMMA was nearly equal to or slightly exceeded that of the free PMMA first extracted, this referring to any of the CA-MA/PMMA samples explored. It follows therefore that there was no appreciable difference in the so-called initiation efficiency between the two polymerization routes stated above.

Taking into consideration the optical and swelling behaviour, too, it may be conceivable for the architecture of the CA-MA/PMMA series that the connected CA-PMMA $(-\mathrm{CA})$ chains and uncross-linked PMMA ones mingled closely with each other to form a network system belonging to a "joined type of IPN," which has been defined previously as an IPN containing some amount of intercomponent cross-links (Miyashita et al. 1996). Homogeneity of the mixing of the polymer components, namely, the compatibility scale in the composite films is discussed below in more detail on the basis of DSC and DMA data.

$T_{\mathrm{g}}$ Evaluation by DSC

DSC measurements were conducted to compare the glass transition behaviour and the degree of compatibility of the polymer components on a dimensional scale less than several tens nanometers (Nishio 1994; Utracki 1990) between the three series of CA/PMMA composites. Figure 3 displays examples of DSC thermograms obtained in the second heating scans for 
film specimens of the composites, together with the corresponding data for CA, PMMA, and CA/PMMA- $b$ films as references. Table 4 compiles $T_{\mathrm{g}}$ values read off as a midpoint of the discontinuity in base-line of the respective thermograms for all the samples explored. $T_{\mathrm{g}} \mathrm{s}$ of CA and PMMA were evaluated as $197.1^{\circ} \mathrm{C}$ and $94.8^{\circ} \mathrm{C}$, respectively. The observation of a somewhat lower $T_{\mathrm{g}}$ value for PMMA, compared with the conventional data $100-110{ }^{\circ} \mathrm{C}$ (Bikson et al. 1994; Brandrup et al. 1999), may be attributable to the essentially lower molecular weight of the polymer product synthesized in this study. $T_{\mathrm{g}} \mathrm{s}$ of CA-MAs were situated in a range of ca. $193-199{ }^{\circ} \mathrm{C}$, bearing a slightly decreasing tendency with increasing degree of mercaptoacetylation (see Table 4). Blend films of the CA/PMMA- $b$ series showed two glass transitions in their thermograms, as illustrated for CA/PMMA-b\#2 in Figure 3a, with reason of the inherent immiscibility of the polymer pair.

\section{$<<$ Figure 3 $>>$}

$<<$ Table 4 >>

Concerning the CA/PMMA-e and CA/PMMA-EG series, any of the composite samples exhibited a dual glass transition behaviour, indicating a phase separation, as did the blends of the CA/PMMA- $b$ series. However, as seen in Figure $3 \mathrm{a}$, the two $T_{\mathrm{g}} \mathrm{s}$ derived from the CA and PMMA components shifted to approach each other in the composite series, relative to the situation in the blend series. For instance, the separation $\Delta T_{\mathrm{g}}$ between the two $T_{\mathrm{g}}$ positions was $83.8^{\circ} \mathrm{C}$ for CA/PMMA- $b \# 2$, and it decreased to $71.1{ }^{\circ} \mathrm{C}$ for CA/PMMA-e\#2 and $64.9{ }^{\circ} \mathrm{C}$ for CA/PMMA-EG ${ }_{0.12} \# 2$; hence, the compatibility of the constituent polymers can be assumed to be better in this order of the sample series, in accordance with the qualitative visual inspection. As for the CA/PMMA-EG series, the diminution in $\Delta T_{\mathrm{g}}$ was more intense with a higher EGDM amount, which is exemplified by a data of $\Delta T_{\mathrm{g}}=53.3{ }^{\circ} \mathrm{C}$ for CA/PMMA-EG ${ }_{0.21} \# 2$, further decreasing from $64.9{ }^{\circ} \mathrm{C}$ for CA/PMMA-EG $0.12 \# 2$. In the present condition of in-feeding for the reagent, undoubtedly, the EGDM cross-linking was fairly effective in promoting interpenetration of polymerized PMMA chains with CA ones and, 
consequently, reduced the scale of phase separation in their composites. In view of these observations, the CA/PMMA-EG series may be taken as being in a class of semi-IPN.

Figure $3 b$ shows a comparison of thermogramic data between different compositions (\#1-3) of the CA-MA/PMMA series; roughly, the ratio CA:PMMA being 20:80 for \#1, 50:50 for \#2, and 80:20 for \#3. We can see an apparently single $T_{\mathrm{g}}$ shifting with the polymer composition. Such a systematic variation in $T_{\mathrm{g}}$ location may imply attainment of a high level of compatibility for this composite series. However, with regard to the two

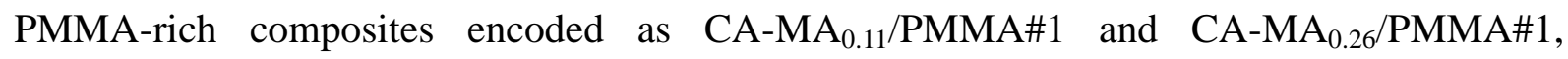
enlargement of their DSC thermograms indicated a feeble, second $T_{\mathrm{g}}$-signal centering $\sim 173{ }^{\circ} \mathrm{C}$ and $\sim 160{ }^{\circ} \mathrm{C}$, respectively (see Table 4). Furthermore, attention should also be paid to the following observations for the \#2-coded compositions. That is to say, the glass transition of CA-MA ${ }_{0.12} / \mathrm{PMMA \# 2}$ stretched over a very wide temperature range, whereas, with another CA-MA of $\mathrm{DS}_{\mathrm{SH}}=0.21$, the transition became narrower and shifted to the lower temperature side, as can be perceived from the corresponding DSC data in Figure $3 \mathrm{~b}$. Accordingly, at least, it is reasonable to assume that the glass transitions of the two constituent polymers were well integrated as an effect of the thiol-ene grafting of PMMA onto CA. A higher frequency of the graft junctions (i.e., the use of CA-MA of a higher $\mathrm{DS}_{\mathrm{SH}}$ ) would be usually advantageous to enhance the integration degree, and to situate the integrated $T_{\mathrm{g}}$ at a lower temperature position, as well.

Concerning the CA-rich composites encoded as \#3, however, the glass transition region was wider in CA-MA ${ }_{0.21} / \mathrm{PMMA \# 3}$ rather than in CA-MA ${ }_{0.12} / \mathrm{PMMA \# 3.} \mathrm{As} \mathrm{demonstrated}$ by Raman spectroscopy in the preceding section (Figure 2), the former sample using CA-MA of such a higher $\mathrm{DS}_{\mathrm{SH}}$ formed disulfide cross-links to a considerable extent among the cellulosic constituent itself. This cross-linking between the semi-rigid chains usually makes the $T_{\mathrm{g}}$ signal broad and indiscernible in the DSC thermogram (Aoki et al. 2007). In the following study of mechanical properties, the \#3-coded compositions are not taken as the 
object, because of the difficulty of separate evaluations of the two contributions, disulfide cross-linking and thiol-ene grafting.

Dynamic Mechanical Analysis

Composite films were examined by DMA to estimate the effect of cross-linking and network formation on their viscoelastic properties and on the mixing state of the components. Figure 4a displays the temperature dependence of $E^{\prime}, E^{\prime \prime}$, and $\tan \delta$ obtained for films of CA and PMMA as references. Both data of $E^{\prime \prime}$ vs. temperature plot make a large dispersion peak centered at ca. $220{ }^{\circ} \mathrm{C}$ (for $\mathrm{CA}$ ) and $90{ }^{\circ} \mathrm{C}$ (for PMMA), which can be associated with the glass transition of the two polymers. A sharp drop of $E^{\prime}$ and a maximum or inflection of $\tan \delta$ are also conspicuous in the respective transition regions. On heating the $\mathrm{CA}$ and PMMA samples above 240 and $140{ }^{\circ} \mathrm{C}$, respectively, the $E$ and $E^{\prime \prime}$ values went out of the measurement limits $\left(\sim 10^{6} \mathrm{Nm}^{-2}\right)$, due to the occurrence of plastic flow of the films.

\section{$<<$ Figure 4 $>>$}

DMA results for \#1 and \#2 composites of the CA/PMMA-e series are shown in Figure 4b. Both samples imparted two dispersion signals in agreement with the double $T_{\mathrm{g}}$ behaviour observed by DSC. The lower-temperature dispersions, providing an $E^{\prime \prime}$ maximum at ca. $110{ }^{\circ} \mathrm{C}$ (for \#1) and $120{ }^{\circ} \mathrm{C}$ (for \#2), are associated with the glass transition of a PMMA-richer phase, and the higher-temperature ones located at ca. $170{ }^{\circ} \mathrm{C}$ (for \#1) and $185^{\circ} \mathrm{C}$ (for \#2) in $E^{\prime \prime}$ correspond to the glass transition of a CA-richer phase. The shifting manner of the dispersion signals from the respective original positions for the PMMA and CA components is also consistent with the result of the DSC study (Table 4).

Figure 4c illustrates DMA data for CA/PMMA-EG ${ }_{0.12} \# 2$ and CA/PMMA-EG $\mathrm{P}_{0.21} \# 2$, both containing equal weight amounts of $\mathrm{CA}$ and PMMA. These composites also gave two 
discrete transitions, but the temperature difference between the locations was generally smaller than that for CA/PMMA-e\#2 (Figure 4b, dotted lines) and the sample CA/PMMA-EG ${ }_{0.21} \# 2$ with a higher content of EGDM signified a closer approach of the two transitions; these are readily found by comparison using the respective $E^{\prime \prime}$ curves. Additionally, the $\tan \delta$ curves above $200{ }^{\circ} \mathrm{C}$ for the CA/PMMA-EG samples were rather depressed without making an upturn toward >1.0, unlike the CA/PMMA-e series. Commonly the ascent in magnitude of $\tan \delta$ beyond 1.0 (i.e., $E^{\prime \prime}>E^{\prime}$ ) implies a transformation of the polymer material concerned into a viscous fluid. The prevention of fluidity observed for the CA/PMMA-EG series is owing to the EGDM cross-linking of the flexible PMMA component.

DMA data for \#1 and \#2 compositions of the CA-MA/PMMA series are illustrated in Figures $4 \mathrm{~d}$ and $4 \mathrm{e}$, respectively; each figure includes a full set of $E, E^{\prime \prime}$, and $\tan \delta$ curves for two samples mutually different in the density of PMMA-branches on the CA trunk component. In the DSC study, the presence of a higher second $T_{\mathrm{g}}$ for the PMMA-rich composites of \#1 was ambiguous (Figure 3b); but, as is proved in Figure 4d, DMA measurements more sensitive to $T_{\mathrm{g}}$ detection (MacKnight et al. 1978) disclosed the corresponding dispersion signal, which was situated in a temperature range $175-195{ }^{\circ} \mathrm{C}$ for $\mathrm{CA}-\mathrm{MA}_{0.11} / \mathrm{PMMA \# 1}$ and in a range $165-185{ }^{\circ} \mathrm{C}$ for $\mathrm{CA}-\mathrm{MA}_{0.26} / \mathrm{PMMA \# 1.} \mathrm{It} \mathrm{is} \mathrm{noteworthy,} \mathrm{however,} \mathrm{that} \mathrm{the} \mathrm{latter}$ sample using CA-MA of a higher $\mathrm{DS}_{\mathrm{SH}}$ showed a considerably gradual decay in the modulus $E$ at high temperatures of $>190^{\circ} \mathrm{C}$, in spite of the PMMA-rich composition.

Two samples of \#2 with a $\sim 50: 50$ composition showed an almost single transition intermediate between 120 and $200{ }^{\circ} \mathrm{C}$, which was followed by appearance of a clear plateau region of $E$ in the side of higher temperatures (Figure 4e). The constant retention of $E$ > $10^{6} \mathrm{Nm}^{-2}$ above $200{ }^{\circ} \mathrm{C}$ reflects a consequence of successful construction of the intercomponent cross-linking network. However, caution should be still exercised to an 
observation for CA-MA ${ }_{0.12} / \mathrm{PMMA \# 2}$; the $E^{\prime \prime}$ curve seems to be composed of an overlap of two dispersion peaks with their maximum at $\sim 140{ }^{\circ} \mathrm{C}$ and at $\sim 180{ }^{\circ} \mathrm{C}$, respectively. This composite sample provided a very broad $T_{\mathrm{g}}$ signal in the DSC measurement (Figure $3 \mathrm{~b}$ ).

In standard DMA studies for binary polymer blends, merging of the principal transitions inherent in the two component polymers ensures the homogeneity of mixing on a scale of less than a couple of tens nanometers (Kaplan 1976; Nishio 1994; Utracki 1990). In the above DMA results, the CA-MA/PMMA\#2 samples substantially satisfy the criterion of such a high level of mixture. As respects the comparison of mixing status between the three series of composites, we can firmly make the rank order of "CA-MA/PMMA (higher) > CA/PMMA-EG > CA/PMMA-e (lower) (> CA/PMMA- $b$ ), " judging from the shifting manner of the corresponding glass transition signals.

Tensile Behaviour

In binary polymer materials, IPN constructions often invite a synergistic improvement in tensile mechanical properties (Nishio 1994; Nishio and Hirose 1992; Thomas and Sperling 1978), far beyond application of the simple additive rule. Acting on the possibility of finding such an IPN effect for the CA/PMMA system, a tensile test was carried out on film specimens of CA, PMMA, and their 50:50 compositions (\#2) of the three network series, under an ambient condition of $23{ }^{\circ} \mathrm{C}$ and $50 \%$ relative humidity. Table 5 collects data of Young's modulus, yield stress (if the yield point was observable), elongation at rupture, and tensile strength. Figure 5 illustrates representative stress-strain curves obtained for CA, PMMA, CA/PMMA-e\#2, CA-MA $0.12 / \mathrm{PMMA \# 2,} \mathrm{and} \mathrm{CA-MA}{ }_{0.21} / \mathrm{PMMA \# 2.}$

\section{$\langle<$ Table 5 >> \\ $<<$ Figure 5 $>>$}

As can be seen from the data in Table 5, composite films of any series showed a higher 
modulus relative to those of plain CA and PMMA films, and the strengths of the composites also surpassed the law of additivity. The generally hard elasticity of the composites comes from the formation of a relatively tight network by virtue of the polymerization process, regardless of the extent of compatibility at the level of a few tens nanometers. Especially, the highest Young's modulus was found for the sample of $\mathrm{CA} / \mathrm{PMMA}-\mathrm{EG}_{0.21} \# 2$, this being indicative of an effectiveness of the chemical cross-linking with EGDM in advancing the film stiffness. However, the operation of only the PMMA component gave rise to no appreciable improvement in toughness against extension of that film.

Of great interest was the tensile behaviour observed for CA-MA/PMMA composites. Film specimens of CA-MA ${ }_{0.12} / \mathrm{PMMA \# 2}$ exhibited an excellent performance in both ductility and tenacity. In the stress-strain curves, a clear yield point appeared at an elongation of 7.5\%, followed by a strain softening region; this is demonstrated in Figure 5. The elongation at rupture and tensile strength of the composite film reached $27.5 \%$ and $56.3 \mathrm{MPa}$, respectively, on average. With regard to the other composite CA-MA $\mathrm{A}_{0.21} / \mathrm{PMMA \# 2}$ having denser cross-linking points between the components, the film specimens were rather less ductile (Figure 5), although they gave absolutely high modulus and strength data (Table 5). It can therefore be inferred that the superiority in tensile property of the former composite CA-MA ${ }_{0.12} / \mathrm{PMMA \# 2}$ was realized by an optimal density of the intercomponent cross-links and entanglements, coupled with the markedly enhanced compatibility of the CA/PMMA pair.

\section{Conclusion}

Three series of IPN-type composites, CA/PMMA-e, CA/PMMA-EG, and CA-MA/PMMA, mutually different in cross-linking situation of the components (Figure 1), were synthesized in film form via polymerization of MMA in a solution system dissolving CA or mercaptoacetylated CA (CA-MA). In preparation of the CA/PMMA-EG series, 
cross-linking of the PMMA component was attained by in-feeding EGDM as cross-linker into the MMA monomer. The CA-MA/PMMA composites were endowed with intercomponent cross-links generated by thiol-ene polymerization.

The mixing state of the two polymer components in the respective series of composites was estimated from observations of the glass transition behaviour by DSC and DMA. With regard to CA/PMMA-e and CA/PMMA-EG composites, a dual glass transition was conspicuous for phase separation; however, two $T_{\mathrm{g}} \mathrm{s}$ associated with the inherently immiscible CA/PMMA pair shifted to approach each other, in contrast with the substantially unmoved situation in the physical blends (CA/PMMA- $b$ series). It can therefore be assumed for those composites that the scale of phase separation was reduced as a result of the chemical blending. The degree of $T_{\mathrm{g}}$ approach was usually more intense in the CA/PMMA-EG series, especially noticeable when prepared with a higher EGDM amount.

The CA-MA/PMMA series, belonging to a joined type of IPN, exhibited a single $T_{\mathrm{g}}$ in DSC measurements, although a certain extent of heterogeneity in phase structure was still perceived for PMMA-rich samples by DMA measurements. In particular, for the compositions of CA:PMMA $=\sim 50: 50$, the mixing scale of less than a couple of tens nanometers was estimated from DMA data showing an almost complete integration of two glass transitions. In a tensile mechanical test, one of the 50:50 composites, having a moderate density of intercomponent linkages, provided an outstanding performance in both ductility and tenacity.

Thus the specific IPN technique using thiol-ene polymerization was found to produce much better compatibility-enhanced CA/PMMA composites. The result of synergistic improvement in mechanical properties also serves as an instance supporting the practical usefulness of this IPN technique in the design of novel polymer composites based on cellulosics. 


\section{References}

Aoki D, Teramoto Y, Nishio Y (2007) SH-Containing cellulose acetate derivatives: preparation and characterization as a shape memory-recovery material. Biomacromolecules 8:3749-3757

Bikson B, Nelson J K, Muruganandam N (1994) Composite cellulose acetate/poly(methyl methacrylate) blend gas separation membranes. J Membrane Sci 94:313-328

Brandrup J, Immergut E H, Grulke E A (1999) Polymer Handbook 4th edn. Wiley, Hoboken, New Jersey

Buchanan C M, Gardner R M, Komarek R J (1993) Aerobic biodegradation of cellulose acetate. J Appl Polym Sci 47:1709-1719

Chang C, Han K, Zhang L (2009) Structure and properties of cellulose/poly( $N$-isopropylacrylamide) hydrogels prepared by IPN strategy. Polym Adv Technol DOI: 10.1002/pat.1616

Edgar K J, Buchanan C M, Debenham J S, Rundquist P A, Seiler B D, Shelton M C, Tindall D (2001) Advances in cellulose ester performance and application. Prog Polym Sci 26:1605-1688

Evans J C, Ellman G L (1959) The ionization of cysteine. Biochim Biophys Acta 33:574-576

Kaplan D S (1976) Structure-property relationships in copolymers to composites: molecular interpretation of the glass transition phenomenon. J Appl Polym Sci 20:2615-2629

Kharasch M S, Read A T, Mayo F R (1938) The peroxide effect in the addition of reagents to unsaturated compounds. XVI. The addition of thioglycolic acid to styrene and isobutylene. Chem Ind 57:752

Landry M R, Massa D J, Landry C J T, Teegarden D M, Colby R H, Long T E, Henrichs P M (1994) A survey of polyvinylphenol blend miscibility. J Appl Poly Sci 54:991-1011

MacKnight W J, Karasz F E, Fried J R (1978) Solid State Transition Behavior of Blends. In: 
Paul D R and Newman S (Eds) Polymer Blends, Vol. 1. Academic Press, New York, Chapter 5, pp 185-242

Miyashita Y, Kimura N, Suzuki H, Nishio Y (1998) Cellulose/poly(acryloyl morpholine) composites: synthesis by solution coagulation/bulk polymerization and analysis of phase structure. Cellulose 5:123-134

Miyashita Y, Nishio Y, Kimura N, Suzuki H, Iwata M (1996) Transition behaviour of cellulose/poly( $N$-vinylpyrrolidone-co-glycidyl methacrylate) composites synthesized by a solution coagulation/bulk polymerization method. Polymer 37:1949-1957

Miyashita Y, Suzuki T, Nishio Y (2002) Miscibility of cellulose acetate with vinyl polymers. Cellulose 9:215-223

Nishio Y (1994) Hyperfine Composites of Cellulose with Synthetic Polymers. In: Gilbert R D (Ed) Cellulosic Polymers, Blends and Composites. Hanser, Munich, Chapter 5, pp $95-113$

Nishio Y (2006) Material functionalization of cellulose and related polysaccharides via diverses microcompositions. Adv Polym Sci 205:97-151

Nishio Y, Hirose N (1992) Cellulose/poly(2-hydroxyethyl methacrylate) composites prepared via solution coagulation and subsequent bulk polymerization. Polymer 33:1519-1524

Ohno T, Yoshizawa S, Miyashita Y, Nishio Y (2005) Interaction and scale of mixing in cellulose acetate/poly( $N$-vinyl pyrrolidone-co-vinyl acetate) blends. Cellulose $12: 281-291$

Ohno T, Nishio Y (2007) Estimation of miscibility and interaction for cellulose acetate and butyrate blends with $N$-vinylpyrrolidone copolymers. Macromol Chem Phys 208:622-634

Thomas D A, Sperling L H (1978) Interpenetrating Polymer Networks. In: Paul D R and Newman S (Eds) Polymer Blends, Vol. 2. Academic Press, New York, Chapter 11, pp $1-33$ 
Utracki L A (1990) Polymer Alloys and Blends: therymodynamics and rheology. Hanser, Munich

Williamson S L, Armentrout R S, Porter R S, McCormick C L (1998) Microstructural examination of semi-interpenetrating networks of poly $(N, N$-dimethylacrylamide $)$ with cellulose or chitin synthesized in lithium chloride/ $N, N$-dimethylacetamide. Macromolecules 31:8134-8141

Yano H, Sugiyama J, Nakagaito A N, Nogi M, Matsuura T, Hikita M, Handa K (2005) Optically transparent composites reinforced with networks of bacterial nanofibers. Adv Mater 17:153-155 


\section{Figure Captions}

Fig 1. Schematic illustrations of different IPN architectures designed for a CA and PMMA pair: (a) CA/PMMA-e entangled mutually without any chemical cross-linkage; (b) CA/PMMA-EG having cross-links only in the vinyl polymer component, and (c) CA-MA/PMMA forming cross-links between the two polymer components

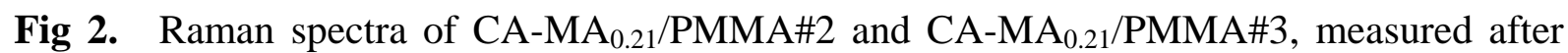
treatment with 2-mercaptoethanol acting as reductant upon disulfide linkage

Fig 3. DSC thermograms obtained in the second heating scan for (a) CA, PMMA, CA/PMMA- $b \# 2$, CA/PMMA-e\#2, and CA/PMMA-EG\#2 samples, and for (b) a series of CA-MA/PMMA samples. Arrows indicate a $T_{\mathrm{g}}$ position taken as the midpoint of the discontinuity in heat flow

Fig 4. Temperature dependence of $E^{\prime}$ (black), $E^{\prime \prime}$ (red), and tan $\delta$ (blue) for CA and PMMA films and their composite ones: (a) data for CA (solid) and PMMA (dotted); (b) data for CA/PMMA-e\#1 (solid) and CA/PMMA-e\#2 (dotted); (c) data for CA/PMMA-EG ${ }_{0.12} \# 2$ (solid) and CA/PMMA-EG $0.21 \# 2$ (dotted); (d) data for CA-MA ${ }_{0.11} / \mathrm{PMMA \# 1} \mathrm{(solid)} \mathrm{and}$

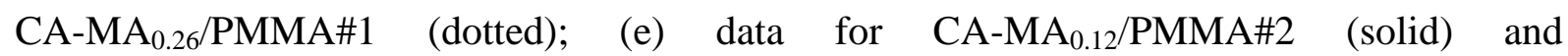

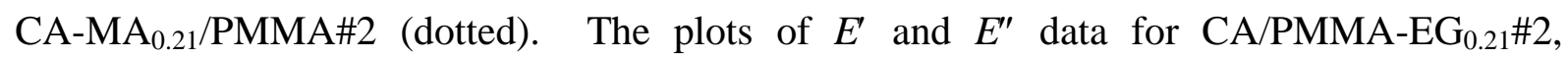

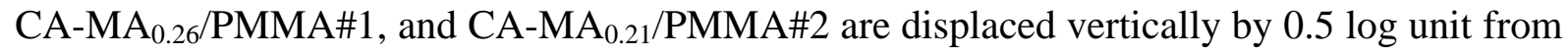
the respective normal positions

Fig 5. Examples of stress-strain curves measured for films of CA, PMMA, CA/PMMA-e\#2, CA-MA ${ }_{0.12} / \mathrm{PMMA \# 2, \text {andCA-MA }}{ }_{0.21} / \mathrm{PMMA \# 2}$ 
In addition to the five figures, there are five tables. See annexed sheets. 
Table 1 Substitution parameters and molecular weights for CA-MA products and the starting CA sample, determined by ${ }^{1} \mathrm{H}$ NMR and GPC, respectively, after propionylation

\begin{tabular}{lccccccc}
\hline Samples & $\mathrm{DS}_{\mathrm{Ac}}$ & $\mathrm{DS}_{\mathrm{SH}}$ & $\mathrm{MS}_{\mathrm{MA}}$ & $\mathrm{DS}_{\mathrm{Pr}}$ & $M_{\mathrm{n}} / 10^{4}$ & $M_{\mathrm{w}} / 10^{4}$ & $M_{\mathrm{w}} / M_{\mathrm{n}}$ \\
\hline $\mathrm{CA}$ & 2.28 & - & - & 0.71 & 6.1 & 19.7 & 3.2 \\
CA-MA $_{0.11}$ & 2.15 & 0.11 & 0.15 & 0.84 & 7.2 & 23.7 & 3.3 \\
CA-MA $_{0.12}$ & 2.17 & 0.12 & 0.20 & 0.83 & 7.1 & 21.4 & 3.0 \\
CA-MA $_{0.21}$ & 2.06 & 0.21 & 0.26 & 0.92 & 6.0 & 20.6 & 3.4 \\
CA-MA $_{0.26}$ & 2.03 & 0.26 & 0.31 & 0.96 & 7.2 & 26.5 & 3.7 \\
\hline
\end{tabular}


Table 2 In-feed amounts of the reagents used for preparation of composite films

\begin{tabular}{|c|c|c|c|c|c|}
\hline \multirow[b]{2}{*}{ Samples } & \multicolumn{3}{|c|}{ CA or } & \multirow{2}{*}{$\begin{array}{c}\text { SH group of } \\
\text { CA-MA/mmol }\end{array}$} & \multirow{2}{*}{$\begin{array}{l}\text { EGDM } \\
/ \mathrm{mmol}\end{array}$} \\
\hline & DMI/g & CA-MA/g & $\mathrm{MMA}^{*} / \mathrm{g}$ & & \\
\hline CA/PMMA-e\#1 & 21.00 & 0.60 & 4.00 & - & - \\
\hline CA/PMMA-e\#2 & 35.00 & 1.00 & 2.08 & - & - \\
\hline CA/PMMA-e\#3 & 56.00 & 1.60 & 1.28 & - & - \\
\hline CA/PMMA-EG ${ }_{0.11} \# 1$ & 21.00 & 0.60 & 4.00 & - & 0.25 \\
\hline $\mathrm{CA} / \mathrm{PMMA}-\mathrm{EG}_{0.26} \# 1$ & 21.00 & 0.60 & 4.00 & - & 0.58 \\
\hline $\mathrm{CA} / \mathrm{PMMA}-\mathrm{EG}_{0.12} \# 2$ & 35.00 & 1.00 & 2.08 & - & 0.45 \\
\hline $\mathrm{CA} / \mathrm{PMMA}-\mathrm{EG}_{0.21} \# 2$ & 35.00 & 1.00 & 2.08 & - & 0.78 \\
\hline CA-MA $0.11 /$ PMMA\#1 & 21.00 & 0.60 & 4.00 & 0.25 & - \\
\hline $\mathrm{CA} \mathrm{MA}_{0.26} / \mathrm{PMMA \# 1}$ & 21.00 & 0.60 & 4.00 & 0.58 & - \\
\hline CA-MA $0.12 /$ PMMA\#2 & 35.00 & 1.00 & 2.08 & 0.45 & - \\
\hline CA-MA ${ }_{0.21} / \mathrm{PMMA \# 2}$ & 35.00 & 1.00 & 2.08 & 0.78 & - \\
\hline $\mathrm{CA} \mathrm{MA}_{0.12} / \mathrm{PMMA \# 3}$ & 56.00 & 1.60 & 0.77 & 0.72 & - \\
\hline $\mathrm{CA} \mathrm{MA}_{0.21} / \mathrm{PMMA \# 3}$ & 56.00 & 1.60 & 0.96 & 1.25 & - \\
\hline
\end{tabular}

${ }^{a}$ Containing DMPA at $0.5 \mathrm{wt} \%$. 
Table 3 Polymer compositions in weight percentage, visual appearance in film form, and molecular weights of the PMMA component estimated by GPC for different series of CA/PMMA samples

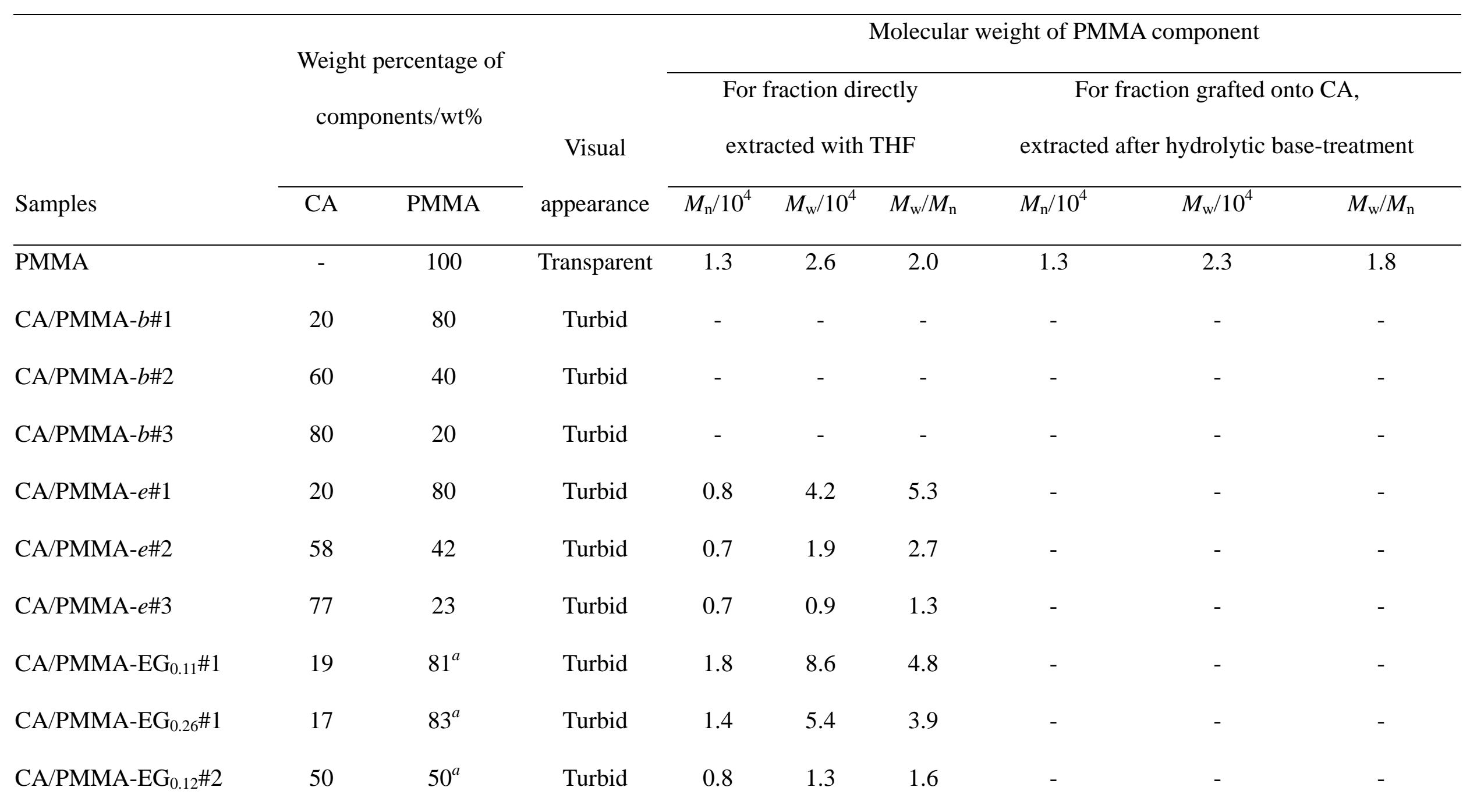




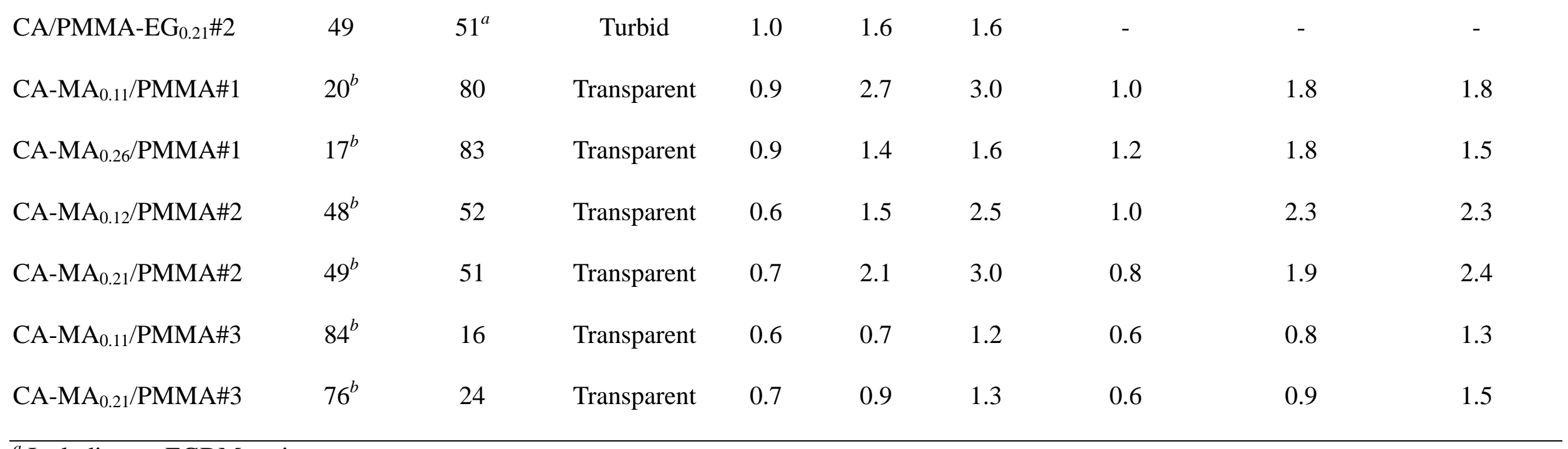

${ }^{a}$ Including an EGDM moiety.

${ }^{b}$ Including a mercaptoacetic acid moiety.

(Table 3, continuation) 
Table $4 T_{\mathrm{g}}$ data evaluated by DSC for CA, CA-MAs, PMMA, and all the series of blends and composites prepared in the present study

\begin{tabular}{|c|c|}
\hline Samples & $T_{\mathrm{g}} /{ }^{\circ} \mathrm{C}$ \\
\hline $\mathrm{CA}$ & 197.1 \\
\hline CA-MA ${ }_{0.11}$ & 199.0 \\
\hline CA-MA ${ }_{0.12}$ & 198.0 \\
\hline CA-MA ${ }_{0.21}$ & 194.3 \\
\hline CA-MA ${ }_{0.26}$ & 192.9 \\
\hline PMMA & 94.8 \\
\hline CA/PMMA- $b \# 1$ & $104.2 / 182.0$ \\
\hline CA/PMMA- $b \# 2$ & $110.1 / 193.9$ \\
\hline CA/PMMA- $b \# 3$ & 106.4 / 195.4 \\
\hline CA/PMMA-e\#1 & $100.2 / 160.0$ \\
\hline CA/PMMA-e\#2 & $101.7 / 172.8$ \\
\hline CA/PMMA-e\#3 & $103.5 / 185.3$ \\
\hline CA/PMMA-EG $0.11 \# 1$ & $105.6 / 161.9$ \\
\hline CA/PMMA-EG $0.26 \# 1$ & $106.6 / 160.6$ \\
\hline CA/PMMA-EG $0.12 \# 2$ & $111.2 / 176.1$ \\
\hline CA/PMMA-EG ${ }_{0.21} \# 2$ & $117.1 / 170.4$ \\
\hline CA-MA ${ }_{0.11} /$ PMMA\#1 & $107.0 /(\sim 173)$ \\
\hline CA-MA ${ }_{0.26} / \mathrm{PMMA \# 1}$ & $95.5 /(\sim 160)$ \\
\hline $\mathrm{CA} \mathrm{MA}_{0.12} / \mathrm{PMMA \# 2}$ & 150.7 \\
\hline CA-MA ${ }_{0.21} /$ PMMA\#2 & 133.4 \\
\hline CA-MA ${ }_{0.12} / \mathrm{PMMA \# 3}$ & 178.5 \\
\hline $\mathrm{CA} \mathrm{MA}_{0.21} / \mathrm{PMMA \# 3}$ & 173.7 \\
\hline
\end{tabular}


Table 5 Tensile mechanical data for films of CA, PMMA, and their $~ 50: 50$ composites

\begin{tabular}{|c|c|c|c|c|}
\hline Samples & $\begin{array}{c}\text { Young's modulus } \\
\text { /GPa }\end{array}$ & $\begin{array}{l}\text { Yield stress } \\
\text { /MPa }\end{array}$ & $\begin{array}{c}\text { Elongation at } \\
\text { rupture/\% }\end{array}$ & $\begin{array}{c}\text { Tensile strength } \\
\text { /MPa }\end{array}$ \\
\hline $\mathrm{CA}$ & $1.47(0.12)^{a}$ & - & $17.0(3.8)^{a}$ & $45.6(3.5)^{a}$ \\
\hline PMMA & $1.45(0.20)$ & - & $1.8(0.1)$ & $23.9(0.4)$ \\
\hline CA/PMMA-e\#2 & $1.97(0.17)$ & - & $4.0(0.4)$ & $43.9(2.7)$ \\
\hline $\mathrm{CA} / \mathrm{PMMA}-\mathrm{EG}_{0.12} \# 2$ & $1.95(0.17)$ & - & $2.1(0.9)$ & $34.6(5.4)$ \\
\hline $\mathrm{CA} / \mathrm{PMMA}-\mathrm{EG}_{0.21} \# 2$ & $2.23(0.25)$ & - & $3.4(0.6)$ & $45.5(1.5)$ \\
\hline CA-MA ${ }_{0.12} / \mathrm{PMMA \# 2}$ & $1.76(0.17)$ & $56.3(2.5)$ & $27.5(1.9)$ & $56.3(5.4)$ \\
\hline $\mathrm{CA} \mathrm{MA}_{0.21} / \mathrm{PMMA \# 2}$ & $1.83(0.25)$ & - & $4.5(0.9)$ & $51.1(1.5)$ \\
\hline
\end{tabular}

${ }^{a}$ Standard deviation is given in parentheses. 

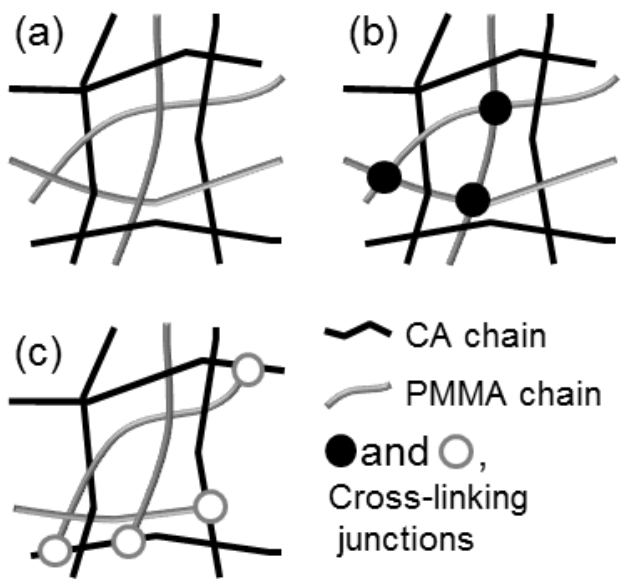

Fig. 1 Schematic illustrations of different IPN architectures designed for a CA and PMMA pair: (a) CA/PMMA-e entangled mutually without any chemical cross-linkage; (b) CA/PMMA-EG having cross-links only in the vinyl polymer component, and (c) CA-MA/PMMA forming cross-links between the two polymer components 


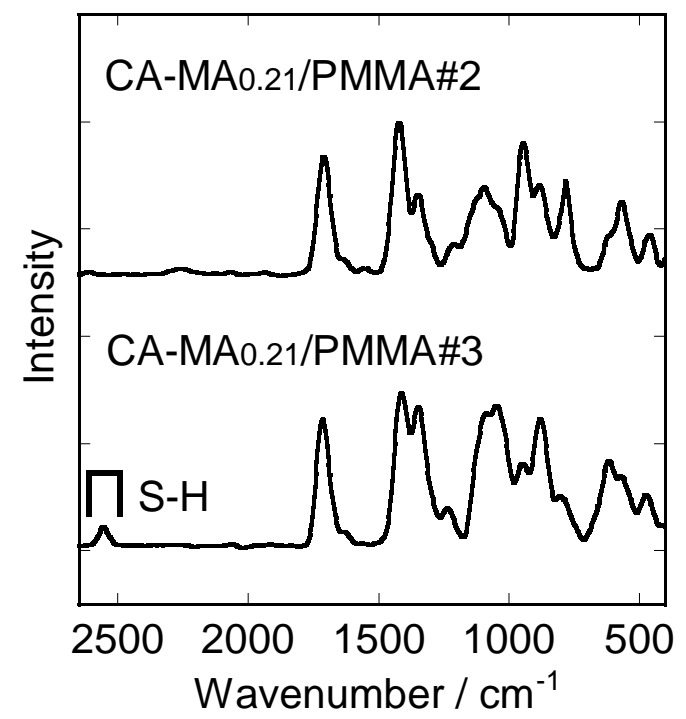

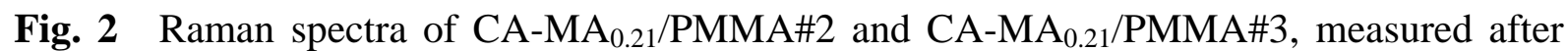
treatment with 2-mercaptoethanol acting as reductant upon disulfide linkage 

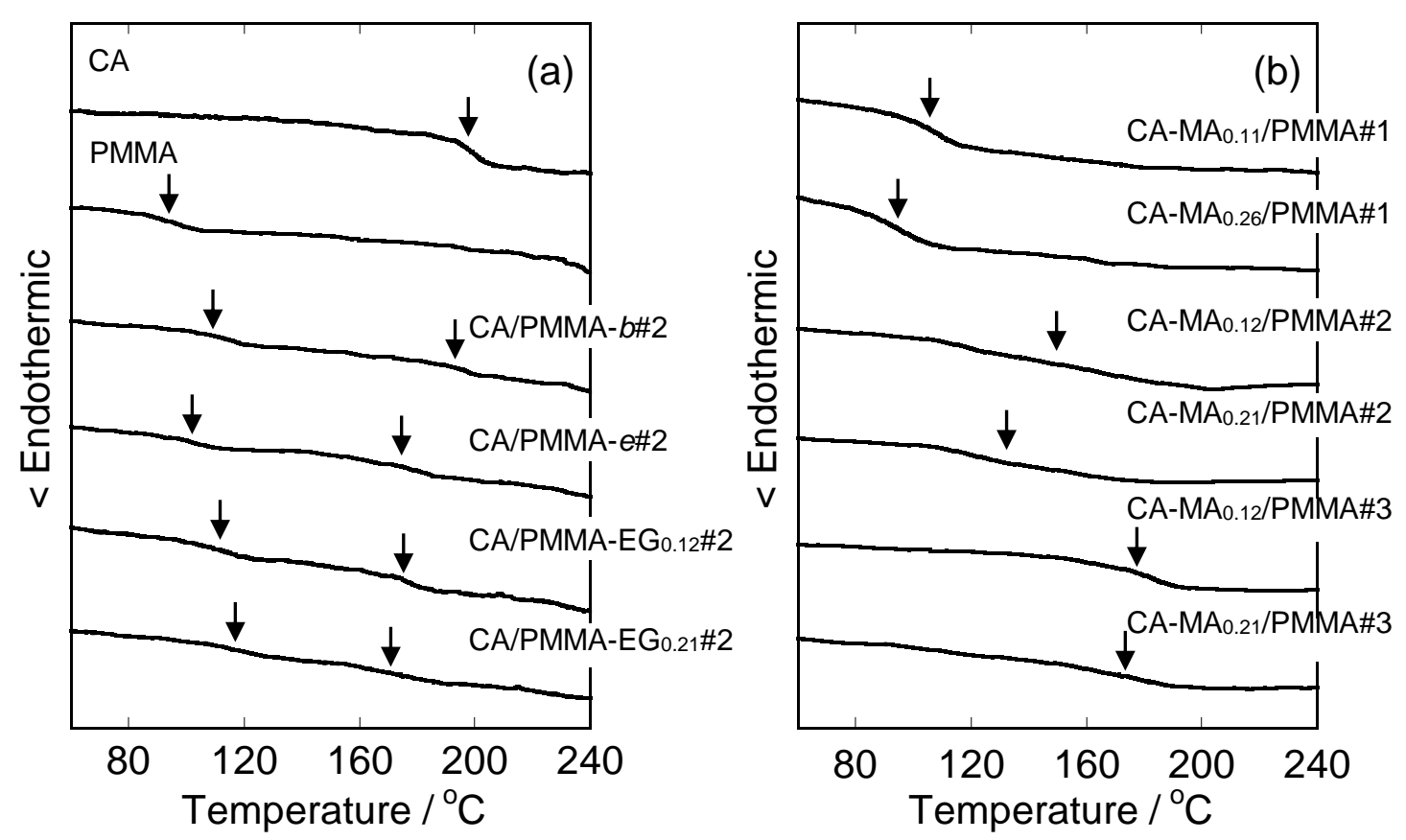

Fig. 3 DSC thermograms obtained in the second heating scan for (a) CA, PMMA, CA/PMMA- $b \# 2$, CA/PMMA- $e \# 2$, and CA/PMMA-EG\#2 samples, and for (b) a series of CA-MA/PMMA samples. Arrows indicate a $T_{\mathrm{g}}$ position taken as the midpoint of the discontinuity in heat flow 

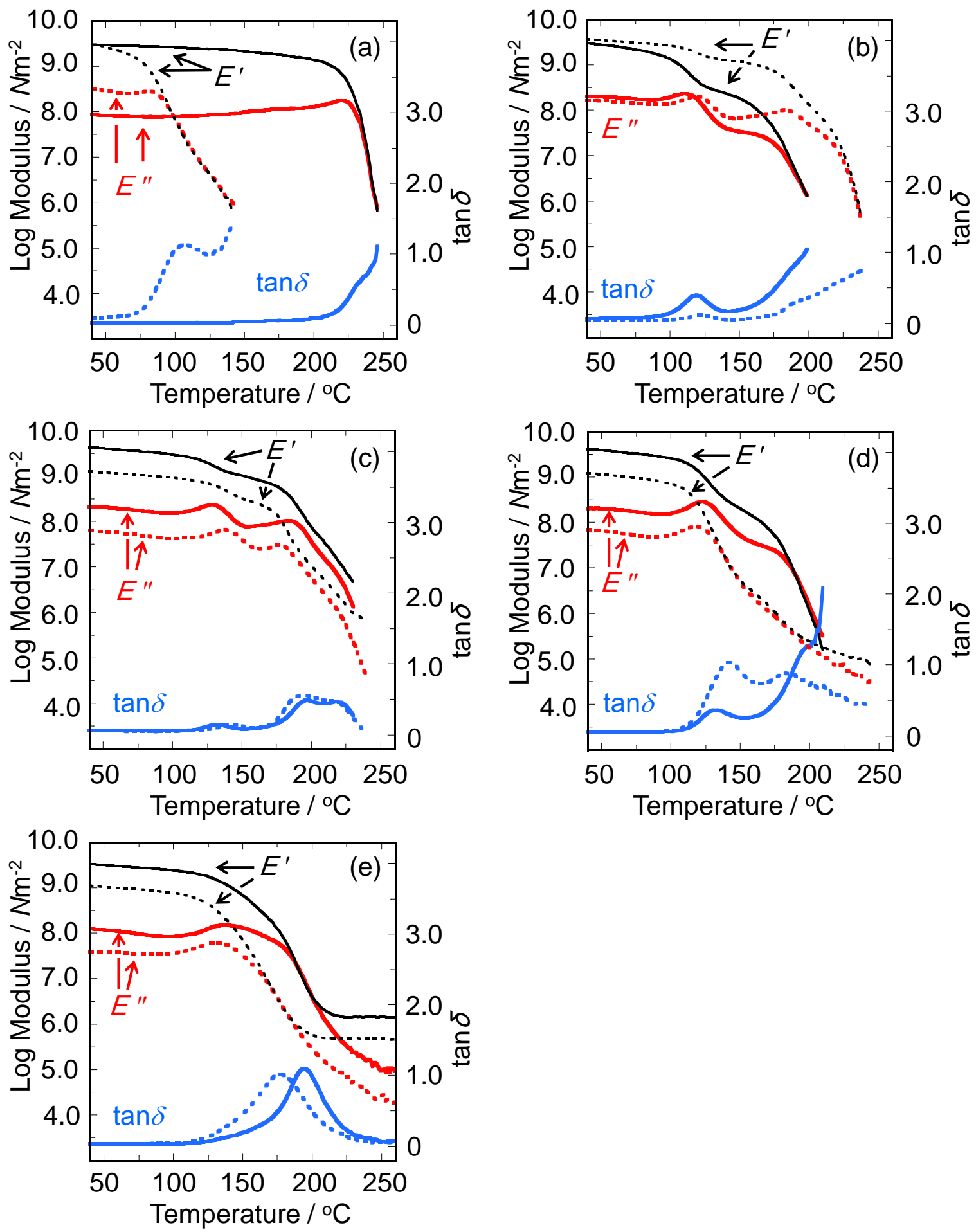

Fig. 4 Temperature dependence of $E^{\prime}$ (black), $E^{\prime \prime}$ (red), and tan $\delta$ (blue) for CA and PMMA films and their composite ones: (a) data for CA (solid) and PMMA (dotted); (b) data for CA/PMMA-e\#1 (solid) and CA/PMMA-e\#2 (dotted); (c) data for CA/PMMA-EG ${ }_{0.12} \# 2$ (solid) and CA/PMMA-EG ${ }_{0.21} \# 2$ (dotted); (d) data for CA-MA ${ }_{0.11} / \mathrm{PMMA \# 1} \mathrm{(solid)} \mathrm{and}$ 


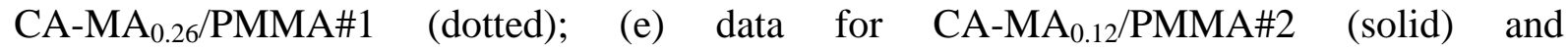
CA-MA $0.21 /$ PMMA\#2 (dotted). The plots of $E$ and $E^{\prime \prime}$ data for CA/PMMA-EG $0.21 \# 2$, CA-MA ${ }_{0.26} / \mathrm{PMMA \# 1}$, and CA-MA ${ }_{0.21} / \mathrm{PMMA \# 2}$ are displaced vertically by $0.5 \log$ unit from the respective normal positions 


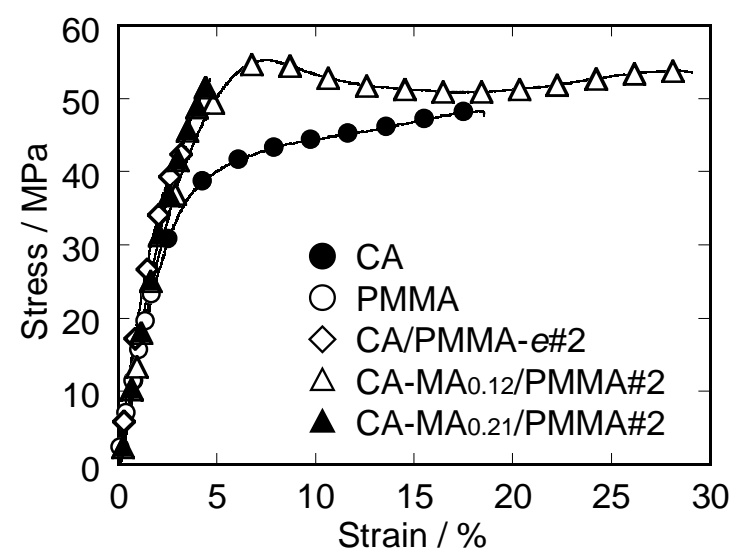

Fig. 5 Examples of stress-strain curves measured for films of CA, PMMA, CA/PMMA-e\#2, CA-MA $0.12 / \mathrm{PMMA \# 2,} \mathrm{and} \mathrm{CA-MA}_{0.21} / \mathrm{PMMA \# 2}$ 